\title{
Gewinnung einer diastatisch wirksamen Substanz aus Milchzucker.
}

\author{
XII. Mitteilung. \\ Von
}

Theodor Panzer.

(Der Redaktion zugegangen am 25. November 1914.)

In früheren Abhandlungen ${ }^{1}$ ) konnte ich zeigen, daß Diastasepräparate, welche durch Aufkochen ihrer Lösung unwirksam gemacht worden sind, in geringem Grade wieder diastatisch wirksam werden, wenn man sie aus der Lösung in festem Zustande abscheidet, dann mit trockenem Chlorwasserstoffgase behandelt und durch länger dauerndes Aufbewahren in luftverdünntem Raume bei Gegenwart einer alkalischen Substanz von Chlorwasserstoff befreit (Auspumpverfahren), oder wenn man die in trockenem Zustande abgeschiedenen Präparate mit trockenem Chlorwasserstoffgas und darauf mit trockenem Ammoniakgas behandelt.

Aus dem anderweitigen Verhalten der "gekochten* Diastasepräparate mußte ich schließen, daß jene Atomgruppe, welche in dem wirksamen Präparate die diastatische Wirkung hervorbringt, aber beim Kochen derart chemisch verändert wird, daß sie diastatisch nicht mehr wirkt, bei der Behandlung mit Chlorwasserstoff gar nicht angegriffen wird.

Wenn dieser Schluß richtig ist und trotzdem durch Einwirkung von Chlorwasserstoff die Entstehung eines wirksamen Diastasepräparates aus einem durch Kochen unwirksam gewordenen angebahnt wird, . so mußte ich vermuten, daß der Chlorwasserstoff eine Atomgruppe angreift, welche bisher mit diastatischer Wirkung überhaupt nichts $\mathrm{zu}$ tun hatte, aber durch das nachfolgende Auspumpen einerseits, die Ammoniakbehandlung anderseits zu einer diastatisch wirksamen Atomgruppe wird. Ich vermutete also, daß durch die genannten Einwirkungen diastatisch wirksame Atomgruppen gewissermaßen

1) Diese Zeitschr., Bd. 86, S. 322 und Bd. 87, S. 115. 
Eine diastatisch wirksame Substanz aus Milchzucker. XIl. 317

neu geschaffen werden, und habe diese Vermutung auch in den zitierten Abhandlungen geäußert.

Ich durfte damals umso eher diese Vermutung äußern, als ich zur selben Zeit schon einen Weg beschritten hatte, der nähere Aufklärung versprach; weil er aus Stoffen von bekannter Konstitution zu Präparaten führte, welche zwar nur geringe, aber trotzdem deutlich erkennbare diastatische Wirkung äußerten.

Ein Zufall hatte mich auf diesen Weg gebracht.

Die aus dem Handel bezogenen Diastasepräparate, welche ich nebst gereinigten Präparaten zu meinen Versuchen verwendet hatte, waren mit Milchzucker vermengt und im Verlaufe der Versuche zeigte es sich, daß diese Beimengung von Milchzucker für die Diastasewirkung nicht gleichgültig war, sie schien im allgemeinen das Wirksamwerden von unwirksam gemachten Diastasepräparaten zu begünstigen. Als ich nun mit den «gekochten» Diastasepräparaten arbeitete, welche nach ihrer Herstellung frei von Milchzucker waren, versuchte ich, das Wiederwirksamwerden dieser Präparate zu steigern, indem ich in verschiedenen Stadien der Versuche Milchzucker beimischte. Da ich es, wie schon seinerzeit bemerkt wurde, für notwendig haltè, alle Versuche tunlichst durch Kontrollversuche $\mathbf{z u}$ bedecken, so habe ich auch hier einen sogenannten blinden Versuch mit reinem Milchzucker angestellt, und da ergab sich das merkwürdige Resultat, daß reiner Milchzucker durch die Behandlung mit trockenem Chlorwasserstoffgas und darauf mit Ammoniakgas zu einem schwach wirkenden Diastasepräparate wurde.

Dieses auffallende Resultat forderte zu einem näheren Studium heraus, dessen erste Ergebnisse in den folgenden Zeilen veröffentlicht werden sollen.

Was die Einzelheiten der Versuchsanordnung betrifft, so verweise ich auf die vorausgegangenen Abhandlungen, deren Angaben ich auch hier genau bis ins kleinste Detail folgte. In großen Zügen möchte ich darüber nun folgendes rekapitulieren.

Eingewogene Proben fein gepulverten Milchzuckers wurden im Vakuumexsikkator über konzentrierter Schwefelsäure bis zum konstanten Gewichte getrocknet, dann in dem seinerzeit 
beschriebenen Apparate durch 24 Stunden hindurch einem langsamen Strome von Chlorwasserstoff ausgesetzt; danach wurde im selben Apparate ein trockener Luftstrom über das Präparat geleitet und dann gewogen. Die prozentische Berechnung des aufgenommenen Chlorwasserstoffs gibt an, wieviel Chlorwasserstoff von. 100 Teilen Milchzucker aufgenommen worden sind, bezw. um wieviel Teile 100 Teile des Präparates an Gewicht zugenommen haben. Nun wurde entweder das «Auspumpverfahren * angeschlossen oder das Präparat wurde in einem ähnlich gebauten Apparate in analoger Weise mit trockenem Ammoniakgas behandelt. Das «Auspumpverfahren * bestand darin, daß das Präparat in einen Vakuumexsikkator kam, der mit konzentrierter Schwefelsäure und mit Natronkalk beschickt war. In der Regel wurde von Tag zu Tag das Gewicht des Präparates festgestellt.

Das fertig gestellte Präparat wurde in Wasser gelöst, die Lösung genau neutralisiert, auf $100 \mathrm{ccm}$ aufgefüllt, mit $100 \mathrm{ccm}$ einer Lösung von löslicher Stärke vermischt und in ein Wasserbad von $50^{\circ} \mathrm{C}$. gebracht. In entsprechenden Zeiten wurden $10 \mathrm{ccm}$ des Gemisches herauspipettiert und darin nach Bang (sog. alte Methode) der Zucker bestimmt. Die nachfolgenden Tabellen geben ohne weitere Umrechnung an, wieviel. Milligramme Zucker (als Traubenzucker gerechnet) in diesen $10 \mathrm{ccm}$ enthalten waren. Gleichzeitig wurde eine weitere herausgenommene Probe des Gemisches mit Jodlösung auf unveränderte Stärke geprüft. Am Schlusse jeden Versuches wurde mit $10 \mathrm{ccm}$ des Restes qualitativ die Fehlingsche Reaktion angestellt. Selbstverständlich wurde auch am Beginne jeden Versuches, d. i. vor dem Einstellen des Gemenges in das Wasserbad, in gleicher Weise der Zuckergehalt bestimmt und die Jodreaktion angestellt. Daß bei den Zuckerbestimmungen das eine Mal $10 \mathrm{ccm}$ einer Flüssigkeit von Zimmertemperatur, das andere Mal $10 \mathrm{ccm}$ einer Flüssigkeit von $50^{\circ} \mathrm{C}$. abpipettiert werden, schließt naturgemäß einen kleinen Fehler in sich, der aber. nur zu ungunsten einer diastatischen Wirkung ausfallen kann, nicht aber zu deren Gunsten und daher keine weitere Rolle spielt. 
Eine diastatisch wirksame Substanz aus Milchzucker. XII. 319

Übrigens wurde in demselben Wasserbade gleichzeitig immer ein Kontrollversuch gehalten, soda $B$ auch in dieser Beziehung ein Fehlschuß vermieden wurde.

Die im folgenden publizierten Resultate beziehen sich hauptsächlich auf Versuche, welche im wesentlichen dartun sollen, daß durch Einwirkung von Chlorwasserstoff und darauf von Ammoniak auf Milchzucker schwach wirksame Diastase entsteht. Nun waren aber aus «gekochter» Diastase nicht nur durch Einwirkung von Chlorwasserstoff und Ammoniak, sondern auch durch Einwirkung von Chlorwasserstoff und Auspumpen wirksame Diastasepräparaten entstanden, und es war daher naheliegend, das letztere Verfahren auch beim Milchzucker zu versuchen. Obwohl sich das Bedürfnis, auch solche Versuche anzustellen, erst im Verlaufe der Arbeit ergeben hat, stelle ich hier diese Versuche an die Spitze.

Einwirkung von Chlorwasserstoff und Auspumpen.

Die in zwei Versuchen festgestellten Gewichtsverhältnisse waren folgende:

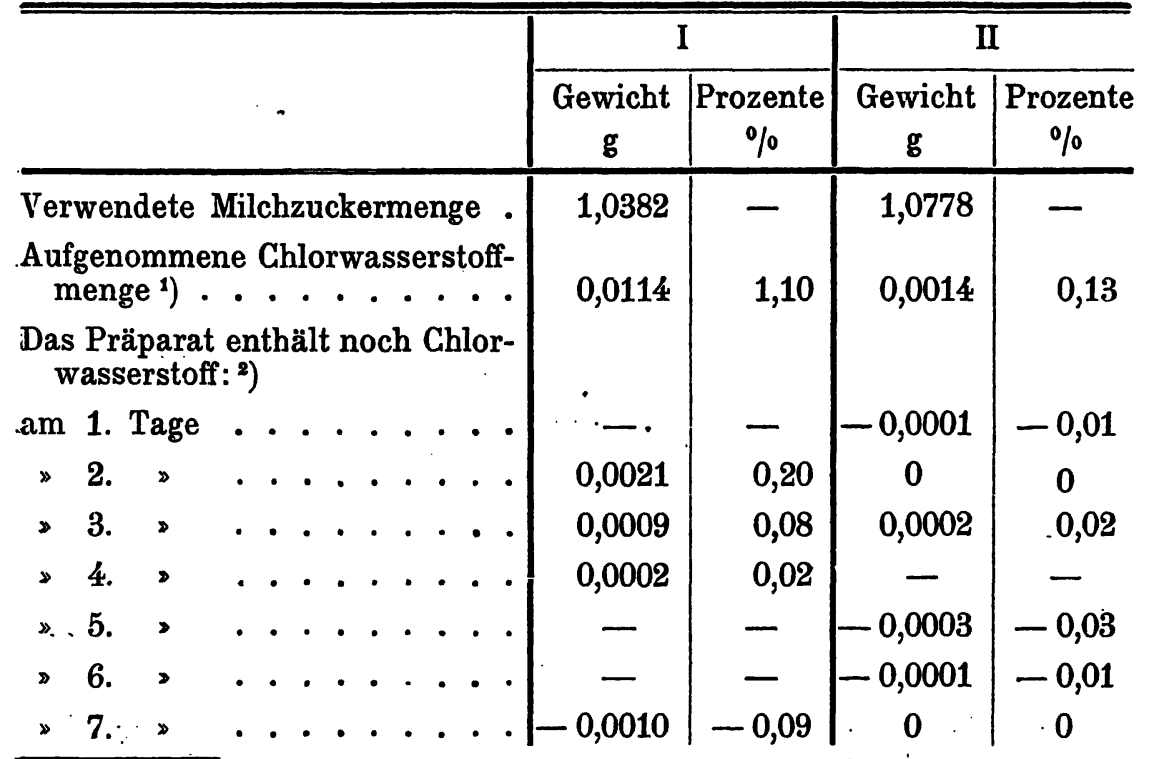

1) Gewichtszunahme.

2) Differenz zwischen dem Gewichte des Präparates an dem betreffenden Tage gegenüber der eingewogenen Milchzuckermenge.

Hoppe-Seyler's Zeitschrift f. physiol. Chemie. XCIII. 
Das Auspumpverfahren wurde noch bis zum 20. Tage fortgesetzt. Das Gewicht beider Präparate sank unter kleinen, wohl als unvermeidliche Wägefehler aufzufassenden Schwankungen noch um eine Kleinigkeit weiter unter das Giewicht des ursprünglich verwendeten Milchzuckers, so daß dieses Minus am 20. Tage betrug:

$$
\begin{gathered}
\text { im I. Versuche: }-0,0017 \mathrm{~g}=-0,16 \% \\
\text { II. } \quad-0,0005 \mathrm{~g}=-0,05 \% .
\end{gathered}
$$

Die Chlorwasserstoffmengen, welche von dem Milchzucker aufgenommen worden sind, sind nicht groß. Beim Auspumpen kehren die Präparate schon in den allerersten Tagen auf ihr ursprüngliches Gewicht zurück, ja sie gehen sogar ein wenig unter ihr ursprüngliches Gewicht herunter. Was dieses Minus $\mathbf{z u}$ bedeuten hat, bezw. ob diese ausgepumpten Präparate von einem Gewichte, welches unter dem Anfangsgewichte liegt, noch chlorhältig sind, und schließlich, ob nur Chlorwasserstoff oder auch noch andere Stoffe bei diesem Auspumpen abgegeben werden, soll Gegenstand späterer Untersuchungen sein.

Die angeführten beiden Präparate wurden nun auf diastatische Wirksamkeit geprüft, die Resultate dieser Versuche geben die beiden folgenden Tabellen:

I.

Kontrolle: 1 g Milchzucker.

\begin{tabular}{|c|c|c|c|c|c|c|}
\hline \multirow{2}{*}{$\begin{array}{l}\text { Dauer des } \\
\text { Versuches }\end{array}$} & \multicolumn{3}{|c|}{ Kontrolle: Milchzucker } & \multicolumn{3}{|c|}{$\begin{array}{c}\text { Mit Chlorwasserstoff } \\
\text { behandelter Milchzucker }\end{array}$} \\
\hline & $\begin{array}{l}\text { Jod- } \\
\text { lösung }\end{array}$ & $\left|\begin{array}{c}\text { Reduktion } \\
\text { in mg } \\
\text { Zucker }\end{array}\right|$ & $\begin{array}{c}\text { mg } \\
\text { Zucker } \\
\text { gebildet }\end{array}$ & $\begin{array}{l}\text { Jod- } \\
\text { lösung }\end{array}$ & $\left|\begin{array}{c}\text { Reduktion } \\
\text { in } \mathrm{mg} \\
\text { Zucker }\end{array}\right|$ & $\begin{array}{c}\text { mg } \\
\text { Zucker } \\
\text { gebildet }\end{array}$ \\
\hline Beginn . • & blau & 35,3 & 0 & blau & 32,8 & 0 \\
\hline 1/2 Stunde . & blau & 34,6 & 0 & blau & 32,5 & 0 \\
\hline 1 & blau & 34,3 & 0 & blau & 31,8 & 0 \\
\hline $11 / 2$ Stunden & blau & 34,9 & 0 & blau & 32,1 & 0 \\
\hline 2 & blau & 34,5 & 0 & blau & 91,9 & 0 \\
\hline $\begin{array}{c}\text { Reduziert } \\
\text { Fehling }\end{array}$ & \multicolumn{3}{|c|}{ sehr stark } & \multicolumn{3}{|c|}{ sehr stark } \\
\hline
\end{tabular}

Stärkelösung: $2 \mathrm{~g}$ lösl. Stärke: $500 \mathrm{ccm}$ Wasser. 
Eine diastatisch wirksame Substanz aus Milchzucker. XIl. 321

II.

Kontrolle: 1 g Milchzucker.

Stärkelösung: $2 \mathrm{~g}$ lösl. Stärke : $500 \mathrm{ccm}$ Wasser.

\begin{tabular}{|c|c|c|c|c|c|c|}
\hline \multirow{2}{*}{$\begin{array}{l}\text { Dauer das } \\
\text { Versuches }\end{array}$} & \multicolumn{3}{|c|}{ Kontrolle: Milchzucker } & \multicolumn{3}{|c|}{$\begin{array}{c}\text { Mit Chlorwasserstoff } \\
\text { behandelter Milchzucker }\end{array}$} \\
\hline & $\begin{array}{l}\text { Jod- } \\
\text { lösung }\end{array}$ & $\begin{array}{c}\text { Reduktion } \\
\text { in mg } \\
\text { Zucker }\end{array}$ & $\begin{array}{c}\text { mg } \\
\text { Zucker } \\
\text { gebildet }\end{array}$ & $\begin{array}{l}\text { Jod- } \\
\text { lösung }\end{array}$ & $\begin{array}{c}\text { Reduktion } \\
\text { in mg } \\
\text { Zucker }\end{array}$ & $\begin{array}{c}\text { mg } \\
\text { Zucker } \\
\text { gebildet }\end{array}$ \\
\hline Beginn . . & blau & 35,2 & 0 & blau & 35,0 & 0 \\
\hline 24 Stunden. & blau & 35,3 & $(0,1)$ & blau & 34,9 & 0 \\
\hline $\begin{array}{c}\text { Reduziert } \\
\text { Fehling }\end{array}$ & \multicolumn{3}{|c|}{ sehr stark } & \multicolumn{3}{|c|}{ sehr stark } \\
\hline
\end{tabular}

Diese Versuche ergeben also ein negatives Resultat. Bei der Einwirkung von Chlorwasserstoff und Auspumpen entsteht aus Milchzucker keine wirksame Diastase, sie entsteht aber, wie die später angeführten Versuche zeigen, dann, wenn Chlorwasserstoff und darnach Ammoniak auf Milchzucker einwirken. Bei den Versuchen mit «gekochter» Diastase waren die Ergebnisse andere. Dort haben beide Verfahren zu wirksamen Diastasepräparaten geführt. Trotzdem stelle ich beide Versuchsreihen in eine Parallele und schließe daraus, daß zum Zustandekommen der für die diastatische Wirksamkeit erforderlichen Atomgruppen Stickstoff notwendig ist. Beim Milchzucker muß dieser Stickstoff erst in Form von Ammoniak zugeführt werden. Die «gekochte» Diastase enthält schon von Hause aus Stickstoff, unter anderem zum Beispiel in Form von Aminogruppen.

Um mit dem eben gezogenen Schlusse nicht mißverstanden $\mathrm{zu}$ werden, betone ich folgendes. Ich habe in meinen bisherigen Arbeiten wiederholt den Ausdruck «diastatisch wirksame Atomgruppe, gebraucht. Unter dieser stelle ich mir eine ziemlich eng begrenzte Atomgruppe vor, an deren Bildung vielleicht nur ein oder zwei Kohlenstoffatome beteiligt sind. Es ist das jene Atomgruppe, von der ich zeigen konnte, daß sie durch Einwirkung von Chlorwasserstoff oder durch Kochen chemisch verändert wird, daß sie durch Einwirkung von Ammoniak nicht angegriffen wird usw. Daß aber nur diese eine Atomgruppe für die diastatische Wirksamkeit erforderlich wäre, 
dafür konnte ich bis jetzt keinerlei Anhaltspunkte gewinnen. Wenn ich nun schließe, daß zum Zustandekommen der für die diastatische Wirksamkeit erforderlichen Atomgruppen Stickstoff notwendig ist, so behaupte ich damit nicht, daß gerade die eben gekennzeichnete «diastatisch wirksame Atomgruppe stickstoffhaltig ist. Es bleibt auch die Möglichkeit offen, daß dieser Stickstoff irgendwo anders im Moleküle der Diastase oder im Sinne der am Beginne des Zyklusses dieser Abhandlungen geäußerten Anschauungen vielleicht sogar in einem ganz anderen chemischen Individuum sitzt.

\section{Einwirkung von Chlorwasserstoff und Ammoniak.}

Ich lasse zunächst einige Protokolle von Versuchen folgen, welche auch zeigen sollen, daß die Versuchsbedingungen variiert wurden.

I.

$1,0250 \mathrm{~g}$ Milchzucker hatten aufgenommen $0,0025 \mathrm{~g}=0,24 \%$ Chlorwasserstoff und darauf $0,0021 \mathrm{~g}=0,20 \%$ Ammoniak, entsprechend einem Überschusse von $0,09 \%$ Ammoniak. ${ }^{1}$ )

Kontrolle: $1 \mathrm{~g}$ Milchzucker.

Stärkelösung: $2 \mathrm{~g}$ lösliche Stärke : $500 \mathrm{ccm}$ Wasser.

\begin{tabular}{|c|c|c|c|c|c|c|}
\hline \multirow{2}{*}{$\begin{array}{l}\text { Dauer des } \\
\text { Versuches }\end{array}$} & \multicolumn{3}{|c|}{ Kontrolle: Milchzucker } & \multicolumn{3}{|c|}{$\begin{array}{l}\text { Mit Chlorwasserstoff und } \\
\text { Ammoniak behandelter } \\
\text { Milchzucker }\end{array}$} \\
\hline & $\begin{array}{l}\text { Jod- } \\
\text { lösung }\end{array}$ & $\begin{array}{c}\text { Reduktion } \\
\text { in mg } \\
\text { Zucker }\end{array}$ & $\begin{array}{c}\text { mg } \\
\text { Zucker } \\
\text { gebildet }\end{array}$ & $\begin{array}{l}\text { Jod- } \\
\text { lösung }\end{array}$ & $\left|\begin{array}{c}\text { Reduktion } \\
\text { in mg } \\
\text { Zucker }\end{array}\right|$ & $\begin{array}{c}\text { mg } \\
\text { Zucker } \\
\text { gebildet }\end{array}$ \\
\hline Beginn . . . & blau & 36,9 & 0 & blau & 34,9 & 0 \\
\hline $1 / 2$ Stunde. & blau & 35,8 & 0 & blau & 34,9 & 0 \\
\hline 1 & blau & 36,2 & 0 & blau & $3 \check{5}, 0$ & 0,1 \\
\hline $1^{1 / 2}$ Stunden & blau & 35,6 . & 0 & blau & $3 \check{0}, 7$ & 0,8 \\
\hline $2 \quad 3$ & blau & 36,0 & 0 & blau & 35,7 & 0,8 \\
\hline $\begin{array}{l}\text { Reduziert } \\
\text { Fehling }\end{array}$ & \multicolumn{3}{|c|}{ sehr stark } & \multicolumn{3}{|c|}{ sehr stark } \\
\hline
\end{tabular}

1) Diese Zahl wurde berechnet, indem von der aufgenommenen Ammoniakmenge $(0,20 \%)$ die zur Neutralisation der aufgenommenen Chlorwasserstoffmenge $(0,24 \%)$ erforderliche Ammoniakmenge $(0,11 \%)$ abgezogen wurde: $0,20-0,11=0,09$. 
Eine diastatisch wirksame Substanz aus Milchzucker. XII. 323

II.

$1,0719 \mathrm{~g}$ Milchzucker hatten aufgenommen $0,0027 \mathrm{~g}=0,25 \%$ Chlorwasserstoff und darauf $0,0010 \mathrm{~g}=0,09 \%$ Ammoniak, entsprechend einem Defizit von $0,03 \%$ Ammoniak.

Kontrolle: $1 \mathrm{~g}$ Milchzucker.

Stärkelösung: $2 \mathrm{~g}$ lösliche Stärke : $500 \mathrm{ccm}$ Wasser.

\begin{tabular}{|c|c|c|c|c|c|c|}
\hline \multirow{2}{*}{$\begin{array}{l}\text { Dauer des } \\
\text { Versuchs }\end{array}$} & \multicolumn{3}{|c|}{ Kontrolle: Milchzucker } & \multicolumn{3}{|c|}{$\begin{array}{l}\text { Mit Chlorwasserstoff und } \\
\text { Ammoniak behandelter } \\
\text { Milchzucker }\end{array}$} \\
\hline & $\begin{array}{l}\text { Jod- } \\
\text { lösung }\end{array}$ & $\left|\begin{array}{c}\text { Reduktion } \\
\text { in mg } \\
\text { Zucker }\end{array}\right|$ & $\underset{\text { Zucker }}{\text { mg }}$ & $\begin{array}{l}\text { Jod- } \\
\text { lösung }\end{array}$ & $\left|\begin{array}{c}\text { Reduktion } \\
\text { in mg } \\
\text { Zucker }\end{array}\right|$ & $\begin{array}{c}\text { mg } \\
\text { Zucker } \\
\text { gebildet }\end{array}$ \\
\hline Beginn . . & blau & 37,5 & 0 & blau & 36,0 & 0 \\
\hline 24 Stunden. & blau & 37,7 & $(0,2)$ & rot & 38,7 & 2,7 \\
\hline $\begin{array}{l}\text { Reduziert. . } \\
\text { Fehling }\end{array}$ & \multicolumn{3}{|c|}{ sehr stark } & \multicolumn{3}{|c|}{ sehr stark } \\
\hline
\end{tabular}

III.

$0,2267 \mathrm{~g}$ Milchzucker hatten aufgenommen $0,0126 \mathrm{~g}=5,56 \%$ Chlorwasserstoff (die Wägung nach der Behandlung mit Ammoniak wurde verabsäumt):

Kontrolle: 0,2 g Milchzucker.

Stärkelösung: $2 \mathrm{~g}$ lösl. Stärke: $500 \mathrm{ccm}$ Wasser.

\begin{tabular}{|c|c|c|c|c|c|c|}
\hline \multirow{2}{*}{$\begin{array}{l}\text { Dauer des } \\
\text { Versuches }\end{array}$} & \multicolumn{3}{|c|}{ Kontrolle: Milchzucker } & \multicolumn{3}{|c|}{$\begin{array}{l}\text { Mit Chlorwasserstoff und } \\
\text { Ammoniak behandelter } \\
\text { Milchzucker }\end{array}$} \\
\hline & $\begin{array}{l}\text { Jod- } \\
\text { lösung }\end{array}$ & $\left|\begin{array}{c}\text { Reduktion } \\
\text { in mg } \\
\text { Zucker }\end{array}\right|$ & $\begin{array}{l}\text { mg. } \\
\text { Zucker } \\
\text { gebildet }\end{array}$ & $\begin{array}{l}\text { Jod- } \\
\text { lösung }\end{array}$ & $\left|\begin{array}{c}\text { Reduktion } \\
\text { in mg } \\
\text { Zucker }\end{array}\right|$ & $\begin{array}{c}\text { mg } \\
\text { Zucker } \\
\text { gebildet }\end{array}$ \\
\hline Beginn . . • & blau & 6,6 & 0 & blau & 5,8 & 0 \\
\hline 1/2. Stunde & blau & 6,5 & 0 & blau & 6,5 & 0,7 \\
\hline 1 & blau & $6,3^{\circ}$ & 0 & blạn & 7,1 & 1,3 \\
\hline 11/2 Stunden & blau & 6,6 & $\mathbf{0}$ & blau & 6,9 & 1,1 \\
\hline $2 \quad$ & blau & 6,2 & 0 & blau & 7,2 & 1,4 \\
\hline $\begin{array}{l}\text { Reduziert } \\
\text { Fehling }\end{array}$ & & stark & & & $1 \mathrm{~K}$ & \\
\hline
\end{tabular}


IV.

$0,2531 \mathrm{~g}$ Michzucker hatten aufgenommen $0,0066 \mathrm{~g}=2,61 \%$ Chlorwasserstoff und darauf $0,0035 \mathrm{~g}=1,34 \%$ Ammoniak, entsprechend einem Überschusse von $0,12 \%$ Ammoniak.

Kontrolle: $0,2 \mathrm{~g}$ Milchzucker.

Stärkelösung: $2 \mathrm{~g}$ lösl. Stärke: $500 \mathrm{ccm}$ Wasser.

\begin{tabular}{|c|c|c|c|c|c|c|}
\hline \multirow{2}{*}{$\begin{array}{l}\text { Dauer des } \\
\text { Versuches }\end{array}$} & \multicolumn{3}{|c|}{ Kontrolle: Milchzucker } & \multicolumn{3}{|c|}{$\begin{array}{l}\text { Mit Chlorwasserstoff und } \\
\text { Ammoniak behandelter } \\
\text { Milchzucker }\end{array}$} \\
\hline & $\begin{array}{l}\text { Jod- } \\
\text { lösung }\end{array}$ & \begin{tabular}{|} 
Reduktion \\
in $\mathrm{mg}$ \\
Zucker
\end{tabular} & $\begin{array}{l}\mathrm{mg} \\
\text { Zucker } \\
\text { gebildet }\end{array}$ & $\begin{array}{l}\text { Jod- } \\
\text { lösung }\end{array}$ & $\left|\begin{array}{c}\text { Reduktion } \\
\text { in mg } \\
\text { Zucker }\end{array}\right|$ & $\begin{array}{c}\text { mg } \\
\text { Zucker } \\
\text { gebildet }\end{array}$ \\
\hline Beginn . . . & blau & 7,1 & 0 & blau & 6,1 & 0 \\
\hline 24 Stunden. & blau & 7,0 & 0 & blau & 7,0 & 0,9 \\
\hline $\begin{array}{l}\text { Reduziert } \\
\text { Fehling }\end{array}$ & \multicolumn{3}{|c|}{ stark } & \multicolumn{3}{|c|}{ stark } \\
\hline
\end{tabular}

Eine Reihe noch weiterer hieher gehöriger Versuche sind von mir als Kontrollversuche $\mathrm{zu}$ anderen Versuchen angestellt worden, sie finden sich im letzten Abschnitte dieser Abhandlung.

In allen diesen Versuchen, mit Ausnahme eines einzigen, zeigt sich bei der Bangschen Titration eine Zunahme des Zuckergehaltes im Sinne einer diastatischen Wirkung. Die genannte Ausnahme (Versuch VII des letzten Abschnittes) ist aber auch dadurch charakterisiert, daß bei der Behandlung mit Chlorwasserstoff so gut wie kein Chlorwasserstoff aufgenommen worden ist. Dieser Versuch war also schon von vorneherein ein Versager, so daß auch keine diastatische Wirkung erwartet werden konnte. Woran es übrigens gelegen war, däß der Milchzucker - und derartiges ist mir einigemale, auch bei anderen Kohlenhydraten, vorgekommen keinen Chlorwasserstoff aufgenommen hat, vermag ich ebensowenig zu sagen, wie das, woran es liegt, daß die aufgenommenen Chlorwasserstoffmengen so verschieden sind. Jedenfalls zeigt sich aber das eine, daß eine Beziehung zwischen der aufgenommenen Chlorwasserstoffmenge und der Intensität der diastatischen Wirkung aus den vorliegenden Versuchen nicht entnommen werden kann. 
Auf den ersten Blick mag vielleicht die Zuckerbildung allzu geringfügig erscheinen. Ich betone, daß die Zuckerwerte der Tabellen sich auf $10 \mathrm{ccm}$ eines Gemenges beziehen, dessen Menge ursprünglich $200 \mathrm{ccm}$ betrug. $1 \mathrm{mg}$ gebildeter Zucker in der Tabelle bedeutet demnach $0,02 \mathrm{~g}$ Zucker, was schon, da das Gemenge 0,4 g löslicher Stärke enthält, ungefähr ein Zwanzigstel jener Zuckermenge ist, welche zu erwarten wäre, wenn die gesamte Stärke vollständig in Zucker umgewandelt wurde.

Nur in zwei Versuchen (Versuch II dieser Reihe und Versuch $\mathrm{V}$ des letzten Abschnittes) gelang es, den Abbau der Stärke bis zum Verschwinden der Stärke zu führen. In den genannten beiden Versuchen zeigt die Reaktion mit Jod an, daß keine Stärke mehr vorhanden war, sondern nur mehr Dextrin. In einem dritten Versuche (Versuch III des letzten Abschnittes) ist dieser Effekt nahezu erreicht, die Jodlösung erzeugte eine violette Färbung.

Es ist eine bekannte Tatsache, daß es in dextrinhaltigen Stärkelösungen mitunter gelingt, durch sehr vorsichtigen Jodzusatz nur die den Dextrinen zukommende Rotfärbung hervorzurufen; erst auf weiteren Zusatz von Jod erscheint die Blaufärbung. Derartige Beobachtungen kann man bekanntlich bei der Jodometrie-nicht selten machen. Diese Tatsache war mir wohlbekannt. Um Mißverständnissen vorzubeugen, betone ich,

1. Daß ich in den herausgegriffenen 3 Versuchen selbstverständlich nicht nach dem ersten Zusatz von einem Tropfen Jodlösung urteilte, sondern darüber hinaus weiter Jod zusetzte.

2. Daß ich wegen der Wichtigkeit der Feststellung in jedem der drei Versuche die Réaktion mit Jod mehrmals anstellte und sie anderen Personen demonstrierte.

3. Daß ich dasselbe Präparat von löslicher Stärke, das zu allen diesen Versuchen gedient hat, seither auch vielfach zu jodometrischen Arbeiten verwendet habe und daß, trotzdem darauf geachtet wurde, niemals die genannte Rotfärbung entstand und ebensowenig durch absichtlich darauf gerichtete Versuche hervorgerufen werden konnte.

Ich kann das vollkommene Verschwinden der Stärke in den beiden genannten Versuchen allerdings nur als glücklichen 
Zufall bezeichnen; denn es gelang mir nicht, durch Variation der Versuchsbedingungen das gleiche Resultat willkürlich hervorzurufen; die diastatische Fähigkeit meiner Präparate ist eben so gering, daß sie leicht "verbraucht» wird.

Was endlich die nur auf qualitativem Wege vorgenommene Prüfung der Flüssigkeiten mit der Fehlingschen Reaktion am Ende jeden Versuches betriff, so wurde sie mehr von einem pedantischen Gesichtspunkte aus vorgenommen, nämlich deshalb, um auch diese Versuche genau ebenso zu gestalten wie die in früheren Abhandlungen publizierten. Diese Reaktion hat im gegebenen Falle wenig praktischen Wert, weil alle aus Milchzucker hergestellten Präparate Fehlingsche Lösung von Hause aus reduzierten. Immerhin war in einigen Fällen, wenn nur wenig (0,2 g) Milchzucker in Arbeit genommen wurde, auch bei der qualitativen Prüfung ein Unterschied gegenüber der Kontrollprobe zu erkennen, ein Plus an Zucker, welches auf Zuckerbildung aus Stärke zurückgeführt werden muß.

In diesem Sinne deuten also alle drei Prüfungen, die Reaktion mit Jodlösung, die quantitative Bestimmung des Zuckers nach Bang und die quantitative Fehlingsche Reaktion auf eine diastatische Wirkung der aus Milchzucker hergestellten Präparate hin.

Einwirkung von Ammoniak, Chlorwasserstoff und Ammoniak auf Milchzucker.

Aus der Erwägung, daß zum Zustandekommen der für die diastatische Wirksamkeit erforderlichen Atomgruppen Stickstoff notwendig ist und daß dieser Stickstoff in den Versuchen mit "gekochter» Diastase schon von vorneherein in den Präparaten enthalten war, entstand der Gedanke, ob es nicht möglich wäre, die Wirksamkeit der aus Milchzucker dargestellten diastatisch wirksamen Präparate zu steigern, wenn man den Milchzucker vor der Behandlung mit Chlorwasserstoff und Ammoniak zuerst stickstoffhaltig macht. Diesem Gedanken entsprechend habe ich Milchzucker zuerst mit Ammoniakgas behandelt und erst dann der Einwirkung von Chlorwasserstoff 
Eino diastatisch wirksame Substanz aus Milchzucker. XII. 327

und wieder von Ammoniak ausgesetzt. Das Ergebnis dicscr Versuche war insofern negativ, als die Wirksamkeit solcher Präparate nicht größer war als in den früher beschricbenen Versuchen. Ich führe nur einen solchen Versuch an.

$0,2260 \mathrm{~g}$ Milchzucker hatton aufgenommen zunuchst keine wilgbaren Mengen von Ammoniak, weiters $0,0120 \mathrm{~g}=5,31 \%$ Chlorwasserstoff, darauf $0,0057 \mathfrak{g}=2,52 \%$ Ammoniak, entsprochond einetn Uberschusse von $0,02 \%$ Ammoniak.

Kontrollo: 0,2 g Milchyuckor.

Starkelరsung: 2 g losl. Starko: $600 \mathrm{ccm}$ Wasser.

\begin{tabular}{|c|c|c|c|c|c|c|}
\hline \multirow{2}{*}{$\begin{array}{l}\text { Daner des } \\
\text { Vorsuches }\end{array}$} & \multicolumn{3}{|c|}{ Kontrolle: Milclızuckor } & \multicolumn{3}{|c|}{$\begin{array}{l}\text { Mit Ammoniak, Chlorwasser- } \\
\text { stoff und Ammoniak } \\
\text { bohandolter Milchzuckor }\end{array}$} \\
\hline & $\begin{array}{l}\text { Jod- } \\
\text { losung }\end{array}$ & $\begin{array}{c}\text { Roduktion } \\
\text { in mg } \\
\text { Zucker }\end{array}$ & $\begin{array}{c}\mathrm{mg} \\
\text { Zuckor } \\
\text { gobildot }\end{array}$ & $\begin{array}{l}\text { Jod- } \\
\text { IJsung }\end{array}$ & \begin{tabular}{|c|} 
Roduktion \\
in ing \\
Zuckor
\end{tabular} & $\begin{array}{l}\text { mg } \\
\text { Zuckor } \\
\text { gobildet }\end{array}$ \\
\hline Beginn . . & blau & 6,5 & 0 & blau & 1,0 & 0 \\
\hline $1 / \times$ Stunde. & blau & 5,6 & 0 & bluu & 5,8 & 0,4 \\
\hline 1. & blau & 6,1 & 0 & blau & 5,6 & 0,7 \\
\hline 11/. Slunden & blau & 5,8 & 0 & bluu & 5,7 & 0,8 \\
\hline $2 \quad$ & blau & 6,0 & 0 & blnu & 6,0 & 1,1 \\
\hline $\begin{array}{c}\text { Reduziorl } \\
\text { Fohling }\end{array}$ & & stark & & & stark & \\
\hline
\end{tabular}

Einfluß der Siedehitze.

Es schien mir nun von Interesse, zu untersuchen, ob dic aus Milchzucker gewonnenen diastatisch wirksamen Präparate sich auch in anderen Belangen so verhalten wie nalürliche Diastase. Derartige Versuche müssen mit einiger Vorsicht vorgenommen worden. Lehrt ja doch die Erfahrung, daß die Eigenschaften, welche gemeinhin als charakteristisch für Fermente angesehen werden, wic Unbestïndigkeit gegen Hitze, Löslichkeit, Fällbarkeit, Temperaturoptimum usw. violfach von der Gegenwart anderer Stoffe beeinflußt werden. In dieser Richtung liegt gerade bei den aus Milchzucker gewonnenen Präparaten die Gefahr eines Fehlschusses nahe, weil in diesen Präparaten kein einziger von jenen Stolfen enthalten ist, welche in der Natur mit tierischen oder pllanzlichen Diastasen vergesellschaftet vorkommen. 
Aus diesem Grunde habe ich vorläufig nur das Verhalten meiner Präparate bei Siedehitze bearbeitet und das Studium anderer Eigenschaften für später verschoben in der Hoffnung, es könnte doch vielleicht inzwischen noch gelingen, wirksamere Präparate auf künstlichem Wege herzustellen.

Die Anordnung der diesbezüglichen Versuche war folgende:

Der eingewogene Milchzucker wurde in der bereits früher angedeuteten Weise mit Chlorwasserstoff und darauf mit Ammoniak behandelt, dann in Wasser gelöst, die Lösung genau neutralisiert und auf $200 \mathrm{ccm}$ aufgefüllt. $100 \mathrm{ccm}$ dieser Flüssigkeit wurden zum Sieden erhitzt und 3 Minuten im Kochen enthalten. Nach dem Abkühlen wurden sie mit $100 \mathrm{ccm}$ Stärkelösung vermischt und zum Versuche verwendet.

Der Rest der neutralisierten Flüssigkeit, also gleichfalls $100 \mathrm{ccm}$, dienten mit $100 \mathrm{ccm}$ derselben Stärkelösung vermischt als Kontrolle. Eine zweite Kontrolle bildeten $100 \mathrm{ccm}$ einer Lösung von unverändertem Milchzucker mit $100 \mathrm{ccm}$ Stärkelösung vermischt.

Die Resultate der in verschiedenen Variationen angestellten Versuche geben folgende Protokolle.

I.

$1,0334 \mathrm{~g}$ Milchzucker hatten aufgenommen $0,0006 \mathrm{~g}=0,06 \%$ Chlorwasserstoff (die Wägung nach der Behandlung mit Ammoniak wurde verabsäumt).

2. Kontrolle: 0,5 g Milchzucker.

Stärkelösung: $2 \mathrm{~g}$ lösl. Stärke: $500 \mathrm{ccm}$ Wasser.

\begin{tabular}{|c|c|c|c|c|c|c|c|c|c|}
\hline \multirow{2}{*}{$\begin{array}{l}\text { Dauer des } \\
\text { Versuches }\end{array}$} & \multicolumn{6}{|c|}{$\begin{array}{l}\text { Mit Chlorwasserstoff und Ammoniak } \\
\text { behandelter Milchzucker }\end{array}$} & \multicolumn{3}{|c|}{$\begin{array}{l}\text { 2. Kontrolle: } \\
\text { Milchzucker }\end{array}$} \\
\hline & $\begin{array}{l}\text { Jod- } \\
\text { lösg. }\end{array}$ & $\begin{array}{c}\text { Reduk- } \\
\text { tion } \\
\text { in mg } \\
\text { Zucker } \\
\end{array}$ & $\underset{\substack{\text { zucker } \\
\text { gebildet }}}{\mathrm{mg}}$ & \begin{tabular}{|l|} 
Jod- \\
lösg.
\end{tabular} & $\begin{array}{c}\text { Reduk- } \\
\text { tion } \\
\text { in mg } \\
\text { Zucker } \\
\end{array}$ & $\left|\begin{array}{c|}\mathbf{m g} \\
\text { zucker } \\
\text { gebildet }\end{array}\right|$ & $\begin{array}{l}\text { Jod- } \\
\text { lösg. }\end{array}$ & $\begin{array}{c}\text { Reduk- } \\
\text { tion } \\
\text { in mg } \\
\text { Zucker } \\
\end{array}$ & $\begin{array}{l}\text { mg } \\
\text { Zucker } \\
\text { gebildet }\end{array}$ \\
\hline Beginn . . & blau & 15,6 & 0 & blau & 16,5 & 0 & blau & 18,9 & 0 \\
\hline $1 / 2$ Stunde & blau & 16,1 & 0,5 & blau & 16,1 & 0 & blau & 18,0 & 0 \\
\hline 1 & blau & 16,6 & 1,0 & blau & 16,1 & 0 & blau & 18,0 & 0 \\
\hline $11 / 2$ Stunden & blau & 16,6 & 1,0 & blau & 16,1 & 0 & blau & 18,4 & 0 \\
\hline 2 & blau & 17,0 & 1,4 & blau & 16,4 & 0 & blau & 18,6 & 0 \\
\hline $\begin{array}{l}\text { Reduziert } \\
\text { Fehling }\end{array}$ & \multicolumn{3}{|c|}{ sehr stark } & \multicolumn{3}{|c|}{ sehr stark } & \multicolumn{3}{|c|}{ sehr stark } \\
\hline
\end{tabular}


Eine diastatisch wirksame Substanz aus Milchzucker. XII. 329

II.

$1,0101 \mathrm{~g}$ Milchzucker hatten aufgenommen $0,0009 \mathrm{~g}=0,09 \%$ Chlorwasserstoff (die Wägung nach der Behandlung mit Ammoniak wurde verabsäumt).

2. Kontrolle: 0,5 g Milchzucker.

Stärkelösung: 2 g lösl. Stärke: $500 \mathrm{ccm}$ Wasser.

\begin{tabular}{|c|c|c|c|c|c|c|c|c|c|}
\hline \multirow{2}{*}{$\begin{array}{l}\text { Dauer des } \\
\text { Versuches }\end{array}$} & \multicolumn{6}{|c|}{$\begin{array}{l}\text { Mit Chlorwasserstoff -und Ammoniak } \\
\text { behandelter Milchzucker }\end{array}$} & \multicolumn{3}{|c|}{$\begin{array}{l}\text { 2. Kontrolle: } \\
\text { Milchzucker }\end{array}$} \\
\hline & $\begin{array}{l}\text { Jod- } \\
\text { lösg. }\end{array}$ & $\begin{array}{l}\text { Reduk- } \\
\text { tion } \\
\text { in } \mathrm{mg} \\
\text { Zucker }\end{array}$ & $\underset{\substack{\text { Zucker } \\
\text { gebildet }}}{\mathrm{mg}}$ & $\begin{array}{l}\text { Jod- } \\
\text { lösg. }\end{array}$ & $\begin{array}{l}\text { Reduk- } \\
\text { tion } \\
\text { in mg } \\
\text { Zucker }\end{array}$ & $\begin{array}{l}\mathrm{mg} \\
\text { zucker } \\
\text { gebildet }\end{array}$ & $\begin{array}{l}\text { Jod- } \\
\text { lösg. }\end{array}$ & $\begin{array}{c}\text { Reduk- } \\
\text { tion } \\
\text { in mg } \\
\text { Zucker }\end{array}$ & $\begin{array}{c}\text { mg } \\
\text { zucker } \\
\text { gebildet } \\
\end{array}$ \\
\hline Beginn . . & blau & 16,0 & 0 & blau & 16,1 & 0 & blau & 19,6 & 0 \\
\hline $1 / 2$ Stunde & blau & 16,3 & 0,3 & blau & 16,1 & 0 & blau & 19,4 & 0 \\
\hline 1 & blau & 16,4 & 0,4 & blau & 15,8 & 0 & blau & 19,5 & 0 \\
\hline $11 / 2$ Stunden & blau & 16,5 & 0,5 & blau & 16,0 & 0 & blau & 19,5 & 0 \\
\hline$>$ & blau & 16,6 & 0,6 & blau & 16,0 & 0 & blau & 19,5 & 0 \\
\hline $\begin{array}{c}\text { Reduziert } \\
\text { Fehling }\end{array}$ & \multicolumn{3}{|c|}{ sehr stark } & \multicolumn{3}{|c|}{ sehr stark } & \multicolumn{3}{|c|}{ sehr star } \\
\hline
\end{tabular}

III.

$1,0426 \mathrm{~g}$ Milchzucker hatten aufgenommen $0,0010 \mathrm{~g}=0,10 \%$ Chlorwasserstoff und darauf Ammoniak in nicht wägbaren Mengen (das Präparat reagiert neutral).

2. Kontrolle: $0,5 \mathrm{~g}$ Milchzucker.

Stärkelösung: $2 \mathrm{~g}$ lösliche Stärke : $500 \mathrm{ccm}$ Wasser.

\begin{tabular}{|c|c|c|c|c|c|c|c|c|c|}
\hline \multirow{3}{*}{$\begin{array}{l}\text { Dauer des } \\
\text { Versuches } \\
\text {. }\end{array}$} & \multicolumn{6}{|c|}{$\begin{array}{l}\text { Mit Chlorwasserstoff und Ammoniak } \\
\text { behandelter Milchzucker }\end{array}$} & \multirow{2}{*}{\multicolumn{3}{|c|}{$\begin{array}{l}\text { 2. Kontrolle: } \\
\text { Milchzucker }\end{array}$}} \\
\hline & \multicolumn{3}{|c|}{$\begin{array}{l}\text { 1. Kontrolle: } \\
\text { ungekocht }\end{array}$} & \multicolumn{3}{|c|}{ geḳocht } & & & \\
\hline & $\begin{array}{l}\text { Jod- } \\
\text { lösg. }\end{array}$ & $\begin{array}{c}\text { Reduk- } \\
\text { tion } \\
\text { in mg } \\
\text { Zucker }\end{array}$ & $\underset{\substack{\text { zucker } \\
\text { gebildet }}}{\mathbf{m g}}$ & $\begin{array}{l}\text { Jod- } \\
\text { lösg. }\end{array}$ & \begin{tabular}{|c|} 
Reduk- \\
tion \\
in $\mathrm{mg}$ \\
Zucker \\
\end{tabular} & $\left|\begin{array}{c}\underset{\text { mg }}{\text { zucker }} \\
\text { gebildet }\end{array}\right|$ & $\begin{array}{l}\text { Jod- } \\
\text { lösg. }\end{array}$ & \begin{tabular}{|c|} 
Reduk- \\
tion \\
in mg \\
Zucker \\
\end{tabular} & $\underset{\substack{\text { Zucker } \\
\text { gebildet }}}{\text { mg }}$ \\
\hline Beginn.. . . & blau & 17,4 & 0 & blau & 17,5 & 0 & blau & 19,5 & 0 \\
\hline 24 Stunden & blau & 18,6 & 1,2 & blau & 17,5 & 0 & blau & 19,5 & 0 \\
\hline $\begin{array}{l}\text { Reduziert } \\
\text { Fehling }\end{array}$ & \multicolumn{3}{|c|}{ sehr stark } & \multicolumn{3}{|c|}{ sehr stark } & \multicolumn{3}{|c|}{ sehr stark } \\
\hline
\end{tabular}


IV.

$0,8509 \mathrm{~g}$ Milchzucker hatten aufgenommen $0,0008 \mathrm{~g}=0,09 \%$ Chlorwasserstoff und darauf $0,0001 \mathrm{~g}=0,01 \%$ Ammoniak (das Präparat reagiert neutral).

2. Kontrolle: $0,5 \mathrm{~g}$ Milchzucker.

Stärkelösung: $2 \mathrm{~g}$ lösliche Stärke : $500 \mathrm{ccm}$ Wasser.

\begin{tabular}{|c|c|c|c|c|c|c|c|c|c|}
\hline \multirow{2}{*}{$\begin{array}{l}\text { Dauer des } \\
\text { Versuches }\end{array}$} & $\begin{array}{l}\text { 1. Kontrolle: } \\
\text { ungekocht }\end{array}$ & \multicolumn{5}{|c|}{$\begin{array}{c}\text { Mit Chlorwasserstoff und Ammoniak } \\
\text { behandelter Milchzucker }\end{array}$} & \multicolumn{3}{|c|}{$\begin{array}{l}\text { 2. Kontrolle: } \\
\text { Milchzucker }\end{array}$} \\
\hline & $\begin{array}{l}\text { Jod- } \\
\text { lösg. }\end{array}$ & $\begin{array}{l}\text { Reduk- } \\
\text { tion } \\
\text { in } \mathrm{mg} \\
\text { Zucker }\end{array}$ & $\begin{array}{c}\mathrm{mg} \\
\text { Zucker } \\
\text { gebildet }\end{array}$ & $\begin{array}{l}\text { Jod- } \\
\text { lösg. }\end{array}$ & $\begin{array}{l}\text { Redak- } \\
\text { tion } \\
\text { in mg } \\
\text { Zucker }\end{array}$ & $\begin{array}{c}\mathrm{mg} \\
\text { Zucker } \\
\text { gebildet }\end{array}$ & $\begin{array}{l}\text { Jod- } \\
\text { lösg. }\end{array}$ & $\begin{array}{c}\text { Reduk- } \\
\text { tion } \\
\text { in mg } \\
\text { Zucker } \\
\end{array}$ & 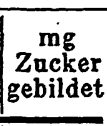 \\
\hline Begin & blau & $17, \mathfrak{5}$ & 0 & blau & 18,0 & 0 & blau & 21,4 & 0 \\
\hline 24 Stunden . & blau & 19,5 & 2,1 & blau & 18,0 & 0 & blau & 21,4 & 0 \\
\hline $\begin{array}{c}\text { Reduziert. } \\
\text { Fehling }\end{array}$ & \multicolumn{3}{|c|}{ sehr stark } & \multicolumn{3}{|c|}{ sehr stark } & \multicolumn{3}{|c|}{ sehr sehr } \\
\hline
\end{tabular}

V.

$0,4348 \mathrm{~g}$ Milchzucker hatten aufgenommen $0,0017 \mathrm{~g}=0,39 \%$ Chlorwasserstoff (die Wägung nach der Behandlung mit Ammoniak wurde verabsäumt).

2. Kontrolle: $0,2 \mathrm{~g}$ Milchzucker.

Stärkelösung: $1 \mathrm{~g}$ lösl. Stärke: $500 \mathrm{ccm}$ Wasser.

\begin{tabular}{|c|c|c|c|c|c|c|c|c|c|}
\hline \multirow{3}{*}{$\begin{array}{l}\text { Dauer des } \\
\text { Versuches }\end{array}$} & \multicolumn{6}{|c|}{$\begin{array}{l}\text { mit Chlorwasserstoff und Ammoniak } \\
\text { behandelten Milchzucker }\end{array}$} & \multirow{2}{*}{\multicolumn{3}{|c|}{$\begin{array}{l}\text { 2. Kontrolle: } \\
\text { Milchzucker }\end{array}$}} \\
\hline & \multicolumn{3}{|c|}{$\begin{array}{l}\text { 1. Kontrolle: } \\
\text { ungekocht }\end{array}$} & \multicolumn{3}{|c|}{ gekocht } & & & \\
\hline & $\begin{array}{l}\text { Jod- } \\
\text { lösg. }\end{array}$ & $\begin{array}{c}\text { Reduk- } \\
\text { tion } \\
\text { in } \mathrm{mg} \\
\text { Zucker } \\
\end{array}$ & $\left|\begin{array}{c|}\text { mg } \\
\text { zucker } \\
\text { gebildet }\end{array}\right|$ & $\begin{array}{l}\text { Jod- } \\
\text { lösg. }\end{array}$ & \begin{tabular}{|c} 
Reduk- \\
tion \\
in $\mathrm{mg}$ \\
Zucker \\
\end{tabular} & $\mid \begin{array}{c}\mathrm{mg} \\
\text { Zucker } \\
\text { gebildet }\end{array}$ & \begin{tabular}{|l|} 
Jod- \\
lösg.
\end{tabular} & \begin{tabular}{|c|} 
Reduk- \\
tion \\
in mg \\
Zucker \\
\end{tabular} & \begin{tabular}{|c}
$\mathrm{mg}$ \\
Zucker \\
gebildet
\end{tabular} \\
\hline eginn . . . & blau & 6,9 & 0 & blau & 7,8 & 0 & blau & 6,5 & 0 \\
\hline 24 Stunden. & rot & 8,0 & 1,1 & blau & 7,8 & 0 & blau & 6,3 & 0 \\
\hline $\begin{array}{c}\text { Reduziert } \\
\text { Fehling }\end{array}$ & \multicolumn{3}{|c|}{ stark } & \multicolumn{3}{|c|}{ mäßig } & \multicolumn{3}{|c|}{ mäßig } \\
\hline
\end{tabular}

VI.

$0,5044 \mathrm{~g}$ Milchzucker hatten aufgenommen $0,0006 \mathrm{~g}=0,12 \%$ Chlorwasserstoff und darauf $0,0009 \mathrm{~g}=0,18 \%$ Ammoniak, entsprechend einem Überschusse von $0,12 \%$ Ammoniak.

2. Kontrolle: 0,2 g Milchzucker.

Stärkelösung: $1 \mathrm{~g}$ lösl. Stärke: $500 \mathrm{ccm}$ Wasser. 
Eine diastatisch wirksame Substanz aus Milchzucker. XII. 331

\begin{tabular}{|c|c|c|c|c|c|c|c|c|c|}
\hline \multirow{2}{*}{$\begin{array}{l}\text { Dauer des } \\
\text { Versuches }\end{array}$} & $\begin{array}{l}\text { Mit Chlorwasserstoff und Ammoniak } \\
\text { behandelter Mlilchzucker }\end{array}$ & $\begin{array}{l}\text { Chlorw } \\
\text { beha } \\
\text { Kontro } \\
\text { angelioc }\end{array}$ & $\begin{array}{l}\text { asserstc } \\
\text { ndelter } \\
\text { lle: } \\
\text { ht }\end{array}$ & $\begin{array}{l}\text { ff un } \\
\text { Alileh }\end{array}$ & $\begin{array}{l}\text { d Amm } \\
\text { zucker } \\
\text { gekock }\end{array}$ & $\begin{array}{l}\text { oniak } \\
\text { t }\end{array}$ & \multicolumn{3}{|c|}{$\begin{array}{l}\text { 2. Kontrolle: } \\
\text { Milchzucker }\end{array}$} \\
\hline & $\begin{array}{l}\text { Jod- } \\
\text { losg. }\end{array}$ & $\begin{array}{l}\text { Reduk- } \\
\text { tion } \\
\text { in mg } \\
\text { Znacker } \\
\end{array}$ & $\begin{array}{c}\text { mg } \\
\text { Zneker } \\
\text { rebildet }\end{array}$ & $\begin{array}{l}\text { Jod- } \\
\text { losg. }\end{array}$ & $\begin{array}{l}\text { Reduk- } \\
\text { tion } \\
\text { in mg } \\
\text { Zucker }\end{array}$ & $\underset{\substack{m g \\
\text { zackebildet } \\
\text { gebild }}}{\mid}$ & $\begin{array}{l}\text { Jod- } \\
\text { loss. }\end{array}$ & $\begin{array}{l}\text { Reduk- } \\
\text { tion } \\
\text { in mg } \\
\text { Zucker } \\
\end{array}$ & $\begin{array}{l}\underset{\mathrm{mg}}{\text { zucker }} \\
\text { gebildet }\end{array}$ \\
\hline Beginn . . & blau & 7,4 & 0 & blau & 7,8 & 0 & blau & 6,0 & 0 \\
\hline 24 Stunden. & blau & 8,2 & 0,8 & blau & 7,7 & 0 & blau & 6,0 & 0 \\
\hline $\begin{array}{c}\text { Reduziert } \\
\text { Fehling }\end{array}$ & \multicolumn{3}{|c|}{ stark } & \multicolumn{3}{|c|}{ mäBig } & \multicolumn{3}{|c|}{ mäßig } \\
\hline
\end{tabular}

VII.

$0,4157 \mathrm{~g}$ Milchzucker hatten aufgenommen $0,0001 \mathrm{~g}=0,02 \%$ Chlorwasserstoff und darauf $0,0015 \mathrm{~g}=0,36 \%$ Ammoniak, entsprechend einem Überschusse von $0,35 \%$ Ammoniak.

2. Kontrolle: $0,2 \mathrm{~g}$ Milchzucker.

Stärkelösung: $1 \mathrm{~g}$ lösl. Stärke: $500 \mathrm{ccm}$ Wasser.

\begin{tabular}{|c|c|c|c|c|c|c|c|c|c|}
\hline \multirow{2}{*}{$\begin{array}{l}\text { Dauer des } \\
\text { Versuches }\end{array}$} & \multicolumn{6}{|c|}{$\begin{array}{l}\text { Mit Chlorwasserstoff und Ammoniak } \\
\text { behandelter Nlilchzucker }\end{array}$} & \multicolumn{3}{|c|}{$\begin{array}{l}\text { 2. Kontrolle: } \\
\text { Milchzucker }\end{array}$} \\
\hline & $\begin{array}{l}\text { Jod- } \\
\text { losg. }\end{array}$ & $\begin{array}{c}\text { Reduk- } \\
\text { tion } \\
\text { in mg } \\
\text { Zucker } \\
\end{array}$ & $\underset{\substack{\text { Zucker } \\
\text { gebildet }}}{\mathrm{mg}}$ & $\begin{array}{l}\text { Jod- } \\
\text { lösg. }\end{array}$ & $\begin{array}{c}\text { Reduk- } \\
\text { tion } \\
\text { in mg } \\
\text { Zucker } \\
\end{array}$ & $\underset{\substack{\text { mg } \\
\text { Zucker } \\
\text { gobildet }}}{\mid}$ & $\begin{array}{l}\text { Jod- } \\
\text { lösg. }\end{array}$ & $\begin{array}{c}\text { Reduk- } \\
\text { tion } \\
\text { in mg } \\
\text { Zucker } \\
\end{array}$ & $\begin{array}{l}\text { mg } \\
\text { Zucker } \\
\text { gebildet }\end{array}$ \\
\hline Beginn . . . & blau & 7,6 & 0 & blau & 7,4 & 0 & blau & 7,9 & 0 \\
\hline $1 / 2$ Stunde & blau & 7,1 & 0 & blau & 7,4 & 0 & blau & 7,5 & 0 \\
\hline 1 & blau & 7,1 & 0 & blau & 7,3 & 0 & blau & 7,6 & 0 \\
\hline 11/2 Stunden & blau & 7,4 & 0 & blau & 7,4 & 0 & blau & 7,7 & 0 \\
\hline 2 & blau & 7,6 & 0 & blau & 7,4 & 0 & blau & 7,6 & 0 \\
\hline $\begin{array}{c}\text { Reduziert } \\
\text { Fehling }\end{array}$ & \multicolumn{3}{|c|}{ mäßig } & \multicolumn{3}{|c|}{ mäßig } & \multicolumn{3}{|c|}{ mäßig } \\
\hline
\end{tabular}

VIII.

$0,4214 \mathrm{~g}$ Milchzucker hatten aufgenommen 0,0004 $\mathrm{g}=0,10 \%$ Chlorwasserstoff und darauf $0,0004 \mathrm{~g}=0,10 \%$ Ammoniak, entsprechend einem Überschusse von $0,05 \%$ Ammoniak.

2. Kontrolle: 0;2 g Milchzucker.

. Stärkelösung: $1 \mathrm{~g}$ lösl. Stärke: $500 \mathrm{ccm}$ Wasser. 


\begin{tabular}{|c|c|c|c|c|c|c|c|c|c|}
\hline \multirow{2}{*}{$\begin{array}{l}\text { Dauer des } \\
\text { Versuches }\end{array}$} & \multicolumn{3}{|c|}{$\begin{array}{l}\text { 1. Kontrolle: } \\
\text { ungekocht }\end{array}$} & \multicolumn{3}{|c|}{ gekocht } & \multicolumn{3}{|c|}{$\begin{array}{l}\text { 2. Kontrolle: } \\
\text { Milchzucker }\end{array}$} \\
\hline & $\begin{array}{l}\text { Jod- } \\
\text { lösg. }\end{array}$ & $\begin{array}{l}\text { Reduk- } \\
\text { tion } \\
\text { in mg } \\
\text { Zucker }\end{array}$ & $\left|\begin{array}{c}\text { mg } \\
\text { Zucker } \\
\text { gebildet }\end{array}\right|$ & $\begin{array}{l}\text { Jod- } \\
\text { lösg. }\end{array}$ & $\begin{array}{l}\text { Reduk- } \\
\text { tion } \\
\text { in mg } \\
\text { Zucker } \\
\end{array}$ & $\begin{array}{c}\mathrm{mg} \\
\text { Zucker } \\
\text { gebildet }\end{array}$ & $\begin{array}{l}\text { Jod. } \\
\text { losg. }\end{array}$ & \begin{tabular}{|c|} 
Reduk- \\
tion \\
in mg \\
Zucker \\
\end{tabular} & $\underset{\begin{array}{l}\text { mg } \\
\text { zucker } \\
\text { gebildet }\end{array}}{\operatorname{mg}}$ \\
\hline Beginn . & blau & 7,4 & 0 & blau & 7,4 & 0 & blau & 8,3 & 0 \\
\hline $1 / 2$ Stunde & blau & 8,1 & 0,7 & blau & 7,3 & 0 & blau & 7,8 & 0 \\
\hline > & blau & 7,6 & 0,2 & blau & 7,4 & 0 & blau & 7,9 & 0 \\
\hline $1^{1} / 2$ Stunden & blau & 7,4 & 0 & blau & 7,4 & 0 & blau & 8,0 & 0 \\
\hline 2 & blau & 8,2 & 0,8 & blau & 7,4 & 0 & blau & 7,9 & 0 \\
\hline $\begin{array}{c}\text { Reduziert } \\
\text { Fehling }\end{array}$ & & mäBig & & & mäl & & & ت & \\
\hline
\end{tabular}

Die angeführten Versuche zeigen, daß diastatisch wirksame Präparate aus Milchzucker beim Kochen ihrer Lösung vollkommen unwirksam werden.

Die aus Milchzucker gewonnenen Diastasepräparate zeigen also auch in dieser Hinsicht dasselbe Verhalten wie natürliche Diastase.

Sind die Anschauungen richtig, welche ich bisher über Enzyme entwickelt habe, dann bedeutet die Gewinnung einer Diastase aus einem Körper von bekannter Konstitution nichts Absonderliches, sie ist nur ein Schritt weiter auf der bereits von mir betretenen Bahn. Geht man aber von den noch herrschenden Ansichten über Enzyme aus, dann müssen die Resultate der oben beschriebenen Versuche wohl als unerwartet bezeichnet werden und man müßte zunächst an alles andere eher denken als an die Neuentstehung eines Enzymes. In diesem Sinne habe ich alle.Einwendungen, die mir auftauchten, wohl geprüft, ehe ich die Resultate dieser Versuche als diastatische Wirkung auffaßte. Eine Reihe solcher Einwände erledigt sich in einfacher Weise durch die Ergebnisse der Versuche von selbst. Es sei mir nur verstattet, hier einige Einwände zu diskutieren, deren Entkräftung einige Überlegung erfordert:

1. Einwand: «Die beobachtete diastatische Wirkung ist Bakterienwirkung» . 
Eine diastatisch wirksame Substanz aus Milchzucker. XII. 333

Ich habe diesen Einwand schon einmal, gelegentlich meiner Versuche mit (gekochter, Diastase behandelt ${ }^{1}$ ) und kann auch für die eben beschriebenen Versuche nur wiederholen, daß es doch höchst sonderbar wäre, wenn sich die niederen Organismen immer nur gerade in jenen Flüssigkeiten entwickeln würden, welche die mit Chlorwasserstoff behandelten Präparate enthielten, während die Kontrollproben davon freigeblieben wären. Man müßte im Gegenteile von der Einwirkung von Chlorwasserstoff eher eine antiseptische Wirkung erwarten.

2. Einwand: «Dem Milchzucker, welcher zu den Versuchen gedient hat, haftete eine kleine Menge Diastase an>.

Dieser Einwand erledigt sich scheinbar ganz einfach durch den Hinweis auf die mit unverändertem Milchzucker angestellten Kontrollversuche, bei welchen auch nicht die geringste Andeutung einer diastatischen Wirkung konstatiert wurde.

Nun könnte man trotz dieser Wirkungslosigkeit doch die Anwesenheit einer geringen Menge von Diastase, aber in ainaktivem» Zustande supponieren und annehmen, daß diese «inaktive Diastase durch Einwirkung von Chlorwasserstoff und Ammoniak in eine «aktive * Form übergeführt wird. Ich gestehe zu, daß eine solche Erklärung meiner Versuche schon sehr weit von dem Wege geraden Denkens abweichen würde, will aber trotzdem auf diesen Einwand eingehen. Ich lasse die Fragen, ob die Milch, aus welcher ja Milchzucker gewonnen wird, überhaupt nennenswerte Mengen von Diastase enthält und ob die Verfahren, welche zur Gewinnung angewendet werden, geeignet sind, etwa vorhandene Diastase zu entfernen, ganz beiseite und betrachte den Einwand als gegeben.

Da ein reiner Milchzucker nur durch mehrfaches Umkrystallisieren gewonnen sein kann, so könnte die kinaktive* Form einer etwa dem Milchzucker anhaftenden Diastase nur eine durch Kochen veränderte Diastase sein. Die «Aktivierung * dieser "gekochten» Diastase durch Behandlung mit Chlorwasserstoff und Ammoniak wäre also dann nichts anderes, als das, was ich in meinen Abhandlungen über *gekochte» Diastase beschrieben habe.

1) Diese Zeitschrift, Bd. 86, $\dot{\text { S. }} 338$. 
Abgesehen davon, daß ich dort das Wiederwirksamwerden von «gekochter» Diastase auf die Neuentstehung von Diastase zurückführen mußte, ergibt sich aus diesen Abhandlungen, daß nur ein geringer Teil der Wirksamkeit der agekochten» Diastase wiederkehrt. Absolut betrachtet ist dieser Teil der wiedergekehrten Wirksamkeit ungefähr so groß wie die Wirksamkeit der aus Milchzucker gewonnenen diastatischen Präparate. Schließt man also zurück, wieviel «gekochte> Diastase der Milchzucker enthalten haben muß, damit der beobachtete Grad von diastatischer Wirksamkeit entstehe, so ergibt sich, daß das Milchzuckerpräparat zur Gänze oder wenigstens nahezu zur Gänze «gekochte» Diastase sein müßte, was natürlich absurd ist.

Immerhin mögen vielleicht bei einer dem Milchzucker anhaftenden «gekochten» Diastase günstigere Umstände obwalten und einen solchen, allerdings schon sehr weit hergeholten Einwand muß ich so lange gelten lassen, als es mir nicht gelingt, diastatische Präparate von größerer Wirksamkeit auf künstlichem Wege zu erhalten.

3. Einwand: «Die beobachtete Zuckerbildung beruht nicht auf einem diastatischen Abbau der Stärke, sondern auf einer allmählich vor sich gehenden Spaltung der Verbindung zwischen Milchzucker und Chlorwasserstoff, bezw. Ammoniak».

Um den Sinn dieses Einwandes näher zu erklären, will ich die durch nichts begründete und mir sogar unwabrscheinlich vorkommende Annahme machen, daß die reduzierende Atomgruppe des Milchzuckers, die Aldehydgruppe, Chlorwasserstoff binde, etwa im Sinne folgender Gleichung:

$$
\mathrm{H}-\mathrm{C}=0+\mathrm{HCl}=\mathrm{HC}<\frac{\mathrm{Cl}}{\mathrm{Cl}}
$$

Der Einwand würde nun besagen, daß durch Einwirkung von Wasser von $50^{\circ} \mathrm{C}$. auf ein solches Reaktionsprodukt die Verbindung mit Chlorwasserstoff allmählich gespalten wird:

$$
\mathrm{HC}_{\mathrm{OH}}^{\mathrm{Cl}}=\mathrm{HCl}+\mathrm{HC}: 0
$$

und daß dadurch die reduzierenden Atomgruppen des Milchzuckers nach und nach wieder hergestellt werden, was na- 
Eine diastatisch wirksame Substanz aus Milchzucker. XII. 335

türlich bei der quantitativen Zuckerbestimmung zum Ausdruck kommen muß.

Eine derartige Erklärung meiner Versuchsergebnisse wird ausgeschlossen durch die Resultate der Versuche des letzten Abschnittes. In diesen Versuchen wurde der mit Chlorwasserstoff und Ammoniak behandelte Milchzucker in Wasser gelöst und die Lösung genau in zwei gleiche Teile geteilt; jeder dieser Teile muß also in diesem Stadium genau dieselbe Reduktionskraft besessen haben.

Die eine Hälfte wird nun mit $100 \mathrm{ccm}$ Stärkelösung vermischt und in den Thermostaten gestellt, die andere Hälfte der Lösung wird zuerst gekocht und dann ebenso behandelt.

Beruht nun die Zuckerbildung, die in der Hälfte der Lösung beobachtet wurde, nur auf dem Zerfall einer Verbindung zwischen Milchzucker und Chlorwasserstoff, bezw. Ammoniak, dann müßte auch die gekochte Hälfte der Lösung ein gleiches Fortschreiten der Reduktionskraft zeigen oder es müßte die gekochte Hälfte, wenn die höhere Temperatur der Siedehitze eine Beschleunigung des Zerfalls verursacht, schon von Beginn des Versuches an eine wesentlich höhere Reduktionskraft aufweisen als die nicht gekochte Hälfte.

Keines von beiden ist der Fall. Allerdings zeigen die gekochten Hälften am Beginne nahezu aller Versuche eine nur wenig höhere Reduktionskraft als die nicht gekochten Hälften, was ja auch ganz erklärlich ist, weil sie beim Kochen durch Eindunsten ein wenig konzentrierter geworden waren, aber nirgends im Verlaufe des Versuches zeigen sie so hohe Reduktionskraft wie die nicht erhitzten Hälften am Ende des Versuches. ${ }^{1}$ )

Der wichtigste Umstand äber, der den diskutierten Einwand ausschließt, ist der, daß in einigen Versuchen durch die Jodreaktion der Abbau der Stärke festgestellt werden konnte.

4. Einwand: "Salzsäure, die durch Zerfall der Verbindung Milchzucker-Chlorwasserstoff frei wird, wirkt hydrolytisch auf Stärke.»

1) Der schon früher diskutierte Versuch VII natürlich ausgenommen. 
Abgesehen davon, daß diese Salzsäuremengen, wie die aufgenommenen Chlorwasserstoffmengen beweisen, nur minimal sein können und überdies, wenigstens zum Teile, beim Neuträlisieren der Flüssigkeit, sowie durch die Produkte der Einwirkung von Ammoniakgas abgestumpft worden sind, erledigt sich dieser Einwand wieder damit, daß die gekochten Lösungen, in welchen eine Hydrolyse eher in höherem Grade erwartet werden müßte, keine Spaltung der Stärke bewirken.

Ich weiß keinen diskutablen Einwand mehr und mus daher dabei bleiben, daß die in dieser Abhandlung beschriebenen Resultate auf der Wirkung einer aus Milchzucker erzeugten Diastase beruhen.

Bemerkungen zu der Arbeit von Kullberg: «Über die gleichzeitige Veränderung des Gehaltes an Glykogen, an Stickstoff und an Enzymen in der Hefe,» Bd. 92, S. 340 dieser Zeitschrift. Von

E. Salkowski.

(Der Redaktion zugegangen am 1. Dezember 1914.) $=$

In einer Arbeit ${ }^{1}$ ): «Über die Bestimmung des Glykogens in der Hefe» habe ich nachgewiesen, daß die von H. Euler ${ }^{2}$ ) benutzte Methode der Glykogenbestimmung in der Hefe von Schönfeld und Krampf bezw. Schönfeld und Künzel notwendig $\mathrm{zu}$ falschen Resultaten führen $\mathrm{muß}$, weil sie eine einfache Anwendung des von Pflüger für tierische Organe angegebenen Verfahrens auf die Hefe ist, bei dem die Besonderheiten der Hefe gegenüber den Organen garnicht berücksichtigt sind.

Ich habe gezeigt, 1. daß das bei diesem Verfahren erhaltene sogenannte Glykogen zu einem großen, ja selbst größten Teil aus Hefegummi besteht, auf welches Pflüger natürlich keine Rücksicht zu nehmen brauchte, 2. daß bei dem Erhitzen der Hefe mit 60\% iger Kalilauge ein Teil der Zell-

1) E. Salkowski, Bd. 92, S. 75 (1914).

2) H. Euler, Über die Rolle des Glykogens bei der Gärung durch lebende Hefe. Diese Zeitschr., Bd. 89, S. 337 (1913) u. Bd. 90, S. 359. 